\title{
Chromosome studies in the aquatic monocots of Myanmar: A brief review with additional records
}

\author{
Yu Ito ${ }^{\dagger}$, Nobuyuki Tanaka ${ }^{\ddagger}$ \\ † University of Canterbury, Christchurch, New Zealand \\ ‡ Kochi Prefectural Makino Botanical Garden, Kochi, Japan
}

Corresponding author: Yu Ito (yu.ito@canterbury.ac.nz), Nobuyuki Tanaka (nbtanaka@makino.or.jp)

Academic editor: Lorenzo Peruzzi

Received: 11 Feb 2014 | Accepted: 08 May 2014 | Published: 13 May 2014

Citation: Ito Y, Tanaka N (2014) Chromosome studies in the aquatic monocots of Myanmar: A brief review with additional records. Biodiversity Data Journal 2: e1069. doi: 10.3897/BDJ.2.e1069

\begin{abstract}
Myanmar (Burma) constitutes a significant component of the Indo-Myanmar biodiversity hotspot, with elements of the Indian, the Indochina, and the Sino-Japanese floristic regions, yet thus far only a few reliable sources of the country's flora have been available. As a part of a contribution for the floristic inventory of Myanmar, since it is important in a floristic survey to obtain as much information as possible, in addition to previous two reports, here we present three more chromosome counts in the aquatic monocots of Myanmar: Limnocharis flava with $2 n=20$, Sagittaria trifolia with $2 n=22$ (Alismataceae), and Potamogeton distinctus $\times P$. nodosus with $2 n=52$ (Potamogetonaceae); the third one is new to science. A brief review of cytological researches in the floristic regions' 45 nonhybrid aquatic monocots plus well investigated two inter-specific hybrids that are recorded in Myanmar is given, indicating that the further works with a focus on species in Myanmar that has infra-specific chromosome variation in the floristic regions will address the precise evolutionary history of the aquatic flora of Myanmar.
\end{abstract}

\section{Keywords}

Aquatic plants, chromosome counts, Limnocharis, Myanmar, Potamogeton, Sagittaria 


\section{Introduction}

With its wealth of plant diversity, Myanmar (Burma) constitutes a significant component of the Indo-Myanmar biodiversity hotspot with elements of the India, the Indochina, and the Sino-Japanese floristic regions (ca, 13,500 vascular plants: Van Dijk et al. 2004; Tanaka 2010). Yet, while neighboring countries' floristic diversity has been exposed through international projects, such as Flora of China, Flore du Cambodge, du Laos et du Vietnam, and Flora of Thailand, thus far no reliable sources of Myanmar's flora have been published except a checklist of spermatophytes contributed by Kress et al. (2003). In order to revise the flora of Myanmar, a decade-long continuous inventory has been conducted by Japanese botanists (Tanaka 2005), which thus far partly contributed a local checklist (Mt. Popa: Tanaka et al. 2006) and a taxon-specific checklist (aquatic plants: Ito and Barfod 2014).

The aim of floristic research is not only to count the total number of species but also to evaluate the native flora's evolutionary origins by comparing with related floristic regions. From this aspect, it is useful to obtain as much information as possible, e.g., chromosome data (Sanders et al. 1983). This is especially important for floristic surveys for aquatic plants, in which infra- or inter-specific chromosome variation is widely known (Les and Philbrick 1993). The proportion of species for which the chromosome number is known is less than $1 \%$ in some little-collected tropical areas (Stace 2000), probably including the southeast Asian country of Myanmar.

Aquatic plants, which is polyphyletically evolved in fern and fern allies, basal angiosperms, monocots, and eudicots, is known as having numerous chromosomal variation, thus an excellent model for this aim. Here, in addition to the previous contributions of chromosome counts for new or noteworthy aquatic plants from Myanmar (Najas tenuis: Ito et al. 2014b; Nechamandra alternifolia: Ito et al. 2009), we present three more chromosome counts for the aquatic monocots of Myanmar: Limnocharis flava (Alismataceae), Sagittaria trifolia (Alismataceae), and Potamogeton distinctus $\times$ P. nodosus (Potamogetonaceae). A brief review of cytological researches in 45 non-hybrid aquatic monocots plus two wellinvestigated inter-specific Potamogeton hybrids in Myanmar is also given with a broad focus on those distributed in neighboring areas, i.e., the Indian, the Indochina, and the Sino-Japanese floristic regions.

\section{Materials and methods}

\section{Chromosome observation}

Plant materials of Limnocharis flava (Alismataceae), Sagittaria trifolia (Alismataceae), Najas tenuis (Hydrocharitaceae), Nechamandra alternifolia (Hydrocharitaceae), and Potamogeton distinctus $\times P$. nodosus (Potamogetonaceae) were collected in the expeditions to Myanmar (Bago Division and Shan State) in 2008. The collections were rigorously identified based on morphological characters using the original protologues as 
well as a previous taxonomic treatment by Cook (1996). Potamogeton distinctus $\times P$. nodosus (Potamogetonaceae) was identified by DNA barcoding method (Ito et al. 2014a). The first set of the voucher specimens was retained in Forest Department Office, Ministry of Environmental Conservation and Forestry, Union of Myanmar (RAF); the duplicates are deposited in two Japanese herbaria: Makino Botanical Garden (MBK) and the University of Tokyo (TI).

Root tips collected in the field were pretreated with $0.002 \mathrm{M}$ 8-hydroxyquinoline at $4{ }^{\circ} \mathrm{C}$ in $12 \mathrm{~h}$, and fixed with freshly mixed Carnoy's fixative (3: 1 ethyl alcohol: acetic acid) for at least $30 \mathrm{~min}$, and then preserved at $4{ }^{\circ} \mathrm{C}$ in $12 \mathrm{~h}$. For microscopic observation, root tips were soaked in $1 \mathrm{~N} \mathrm{HCl}$ for $1 \mathrm{~h}$ followed by $10 \mathrm{~min}$ at $60{ }^{\circ} \mathrm{C}$. After being immersed in tap water, the materials were stained in a drop of $1.5 \%$ orcein acetate solution on a slide glass in 5 min., and then squashed. Then somatic chromosome numbers of the three taxa were obtained by light microscopic examination. For each species, at least two cells were used to confirm the numbers.

Distribution for each species follows Ito and Barfod (2014).

\section{Literature review}

Chromosome researches for aquatic monocots of Myanmar were reviewed with a broad focus on Myanmar and related floristic regions, i.e., the Indian, the Indochina, and the Sino-Japanes floristic regions. The focal species include 45 non-hybrid aquatic monocots listed in Ito and Barfod (2014), Ito et al. (2014a) as well as well-investigated two interspecific Potamogeton hybrids (Ito et al. 2014a). Initial literature search was carried out with Fedorov (1969) as well as Index to Plant Chromosome Numbers (Missouri Botanical Garden, http://mobot.mobot.org/W3T/Search/ipcn.html), followed by extensive literature review with original references. For some species, mostly cosmopolitan ones, only a few representative literature references are given for each chromosome number. Since a comprehensive cytological review was given for aquatic plants (Les and Philbrick 1993), including almost all the taxa listed in the present study, our literature review focused on literature published in 1993 or later. Due to incapability of original references, some rare chromosome counts are not included; those references are mostly published in 1970 or earlier, and written not in English. No detailed references are given for Potamogetonaceae and Ruppia because an exhaustive cytological review was published by Kaplan et al. (2013), Talavera et al. (1993). 


\section{Chromosome counts for the aquatic monocots of Myanmar}

\section{Order Alismatales}

\section{Family Alismataceae}

Genus Limnocharis Bonpl., 1808

Limnocharis flava (L.) Buchenau, 1868

Material

a. country: Myanmar; stateProvince: Bago; municipality: Pyat Township; locality: along the roadside, paddy field, ca. $30 \mathrm{~km}$ east of Pyat; verbatimLatitude: $18^{\circ} 49^{\prime} 44^{\prime \prime} \mathrm{N}$;

verbatimLongitude: 95¹8'06"E; eventDate: 7 Dec 2008; recordedBy: Y. Ito; collectionID:

N. Tanaka \& al. 080776; institutionCode: MBK, RAF, TI

Distribution: Native to Americas; naturalized to tropical Asia.

Notes: Chromosome counts: $2 n=20$ (Fig. 1; obtained in this study).

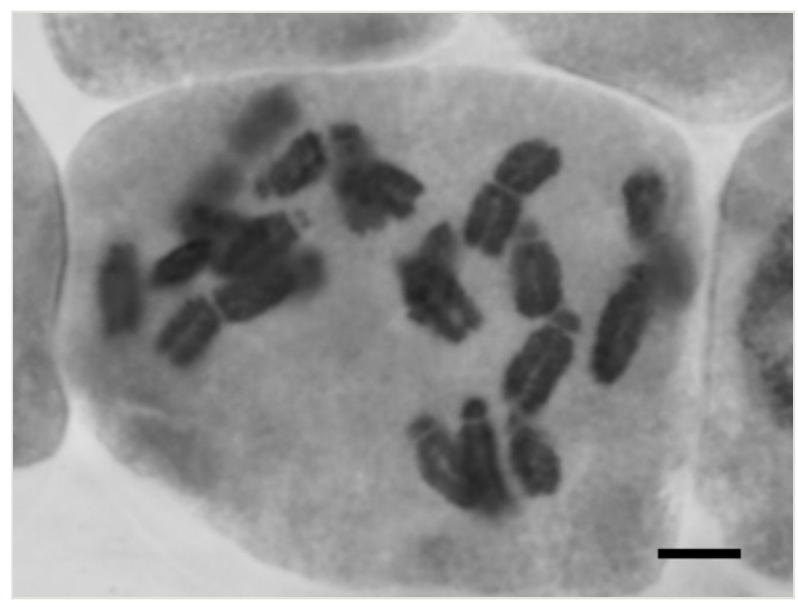

Figure 1.

Somatic chromosome of Limnocharis flava. Bar indicates $5 \mu \mathrm{m}$. 
Genus Sagittaria L., 1753

\section{Sagittaria trifolia L., 1753}

\section{Material}

a. country: Myanmar; stateProvince: Shan; verbatimLocality: Pindaya; verbatimLatitude: $20^{\circ}$ 59'57"N; verbatimLongitude: 96³9'59"E; eventDate: 1 Dec 2008; recordedBy: Y. Ito; collectionID: N. Tanaka \& al. 080623

Distribution: Bangladesh, Bhutan, China (nationwide), India (nationwide), Indonesia (Borneo, Java, Sulawesi), Japan, Malaysia (Peninsular), Myanmar, Nepal, Pakistan, Philippines, Thailand; Oceania.

Notes: Chromosome counts: $2 n=22$ (Fig. 2; obtained in this study).

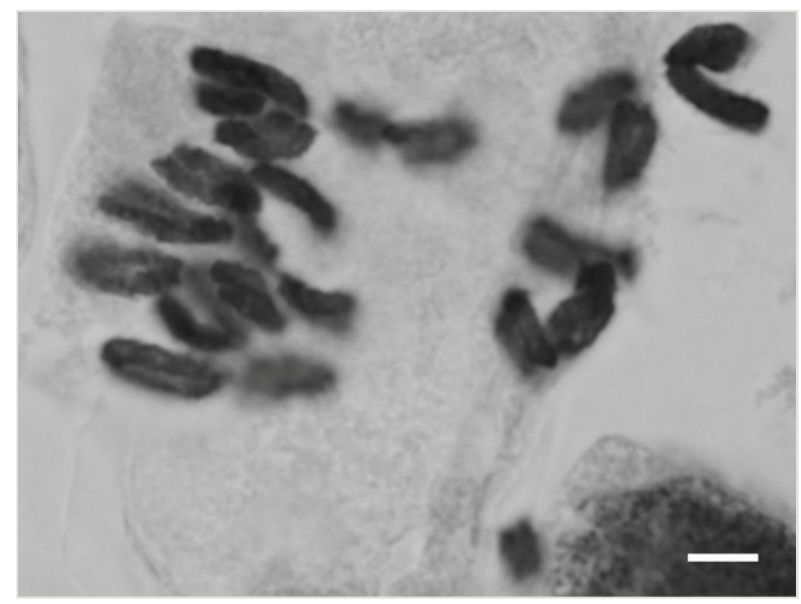

Figure 2.

Somatic chromosome of Sagittaria trifolia. Bar indicates $5 \mu \mathrm{m}$. 


\section{Family Hydrocharitaceae}

\section{Genus Najas L., 1753}

\section{Najas tenuis Magnus, 1870}

\section{Material}

a. $\quad$ country: Myanmar; stateProvince: Shan; verbatimLocality: Inlay Lake, Nyaung Shwe Township; verbatimLatitude: $20^{\circ} 32^{\prime} 02^{\prime \prime} \mathrm{N}$; verbatimLongitude: $96^{\circ} 53^{\prime} 53 " \mathrm{E}$; eventDate: 3 Dec 2008; recordedBy: Y. Ito; collectionID: N. Tanaka \& al. 080642; institutionCode: MBK, RAF, TI

Distribution: India (Central, Southern), Myanmar, Sri Lanka.

Notes: Chromosome counts: $2 n=24$ (Fig. 3; After Ito et al. 2014b; reproduced with publisher's permission).

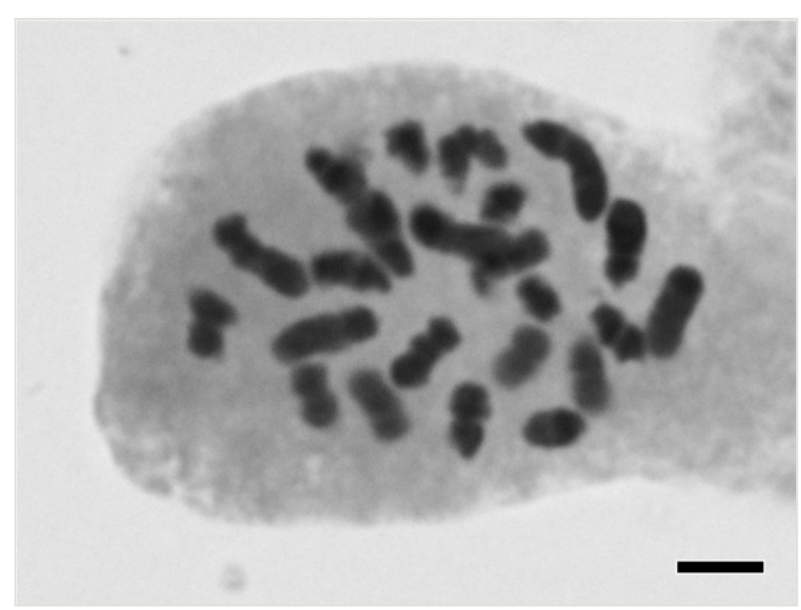

\section{Figure 3.}

Somatic chromosome of Najas tenuis. Bar indicates $5 \mu \mathrm{m}$. 
Genus Nechamandra Planch., 1849

Nechamandra alternifolia (Roxb.) Thwaites, 1864

Material

a. $\quad$ country: Myanmar; stateProvince: Shan; verbatimLocality: Near Yae Aye Kan Dam, Yae Aye Kan, Kalaw Township; verbatimLatitude: $20^{\circ} 35^{\prime} 37^{\prime \prime N}$; verbatimLongitude: 96³1'46"E; eventDate: 26 Nov 2008; recordedBy: Y. Ito; collectionID: N. Tanaka \& al. 080058; institutionCode: MBK, RAF, TI

Distribution: Bangladesh, China (Southern), India (Eastern, Northern, Southern), Myanmar, Nepal, Sri Lanka, Thailand, Vietnam; Yemen, and Sudan.

Notes: Chromosome counts: $2 n=16$ (Fig. 4; After Ito et al. 2009; reproduced with publisher's permission).

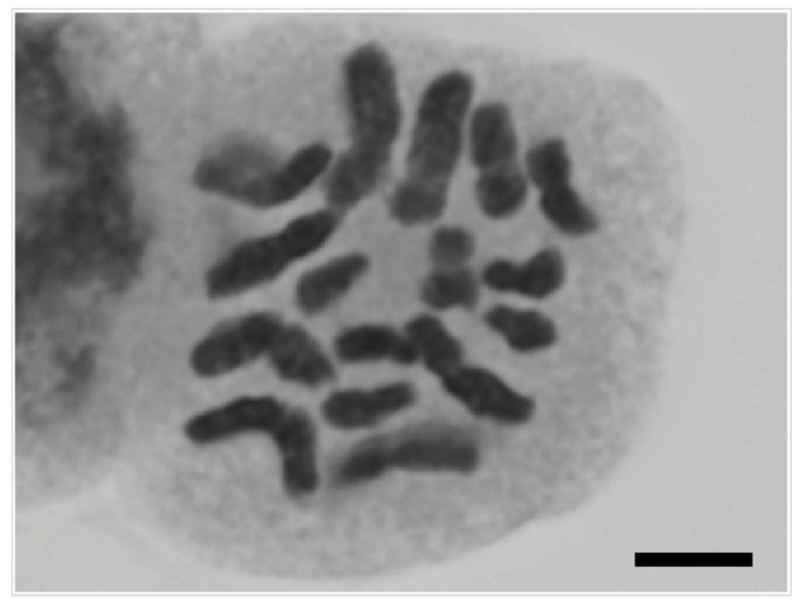

Figure 4.

Somatic chromosome of Nechamandra alternifolia. Bar indicates $5 \mu \mathrm{m}$.

\section{Family Potamogetonaceae}

Genus Potamogeton L., 1753

Potamogeton distinctus A. Benn. × P. nodosus Poir.

Material

a. country: Myanmar; stateProvince: Shan; verbatimLocality: Inle Lake; verbatimLatitude: 20²7'28"N; verbatimLongitude: 9650'37"E; eventDate: 4 Dec 2008; recordedBy: Y. Ito; collectionID: N. Tanaka \& al. 080662; institutionCode: MBK, RAF, TI 
Notes: Chromosome counts: $2 n=52$ (Fig. 5 ; obtained in this study). The chromosome count for this taxon is new to science.

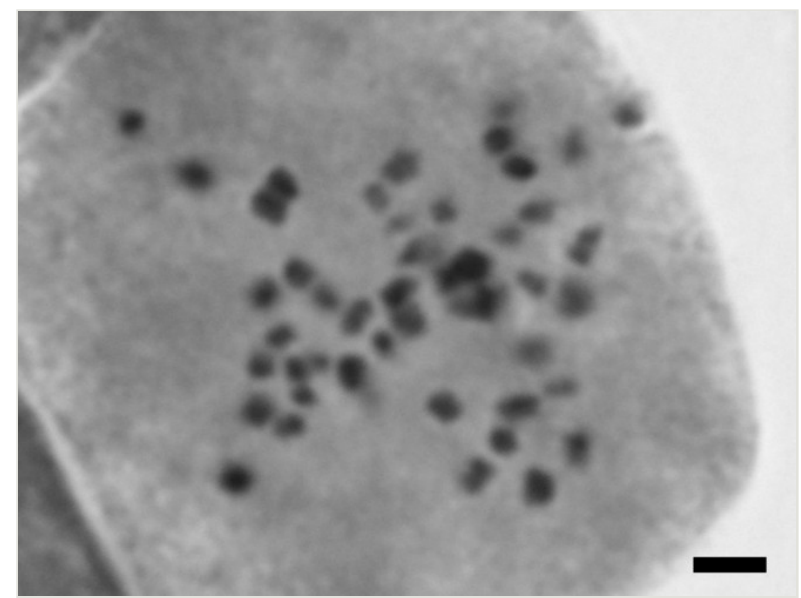

Figure 5.

Somatic chromosome of Potamogeton distinctus $\times$ P. nodosus. Bar indicates $2.5 \mu \mathrm{m}$.

\section{Analysis}

The chromosome counts given for 45 non-hybrid species of aquatic monocots of Myanmar as well as well-investigated two Potamogeton hybrids among them were reviewed with a focus on infra-specific chromosome variation (Table 1). The cited literature references also include chromosome counts obtained from related floristic regions, i.e., the Indian, the Indochina, and the Sino-Japanese floristic regions. For widespread species, cytological information from other regions is cited. 
Table 1.

The chromosome counts given for 45 non-hybrid species of aquatic monocots of Myanmar as well as well-investigated two Potamogeton hybrids among them. Those recorded from neighboring regions are also provided. The species that have no chromosome counts anywhere in the world are shown with n/a. For some species, mostly cosmopolitan ones, only a few representative literature references are given for each chromosome number. Note that due to incapability of original references, some rare chromosome counts are not included in this table: $2 n=18,42,48$ for Acorus calamus; $2 n=18,22$ for Acorus gramineus (Acoraceae), $2 n=28$ for Pistia stratiotes var. cuneata Engl.; $2 n=28$ for Pistia stratiotes var. spathulata (Michx.) Engl.; $2 n=20,50,60,80$ for Lemna aequinoctialis; $2 n=44$ for Lemna trisulca; $2 n=30,50$ for Spirodela polyrrhiza (Araceae); $n=14$ $(2 n=28), 2 n=10,12$ for Alisma plantago-aquatica; $2 n=22$ for Caldesia parnassifolia; $2 n=26,39$ for Limnocharis flava; $2 n=22$ for Sagittaria trifolia var. longiloba (Turr.) Mak.; $2 n=22$ for Sagittaria trifolia var. sinensis Sims; $2 n=22$ for Sagittaria trifolia var. edulis (Sieb.) Ohwi (Alismataceae); $2 n=$ 24 for Blyxa aubertii; $2 \mathrm{n}=60$ for Najas marina; $2 \mathrm{n}=12+1 \mathrm{~B}$ for Najas marina var. intermedia (Gorski) A. Braun; $2 n=22,52,72,88,132$ for Ottelia alismoides; $2 n=16,22,28,33$ for Vallisneria spiralis (Hydrocharitaceae); $2 n=64$ for Eichhornia crassipes; $2 n=26, n=40(2 n=80)$ for Monochoria vaginalis (Pontederiaceae); $2 n=60$ for Typha angustifolia (Typhaceae). Also refer to previous cytological reviews (aquatic plants: Les and Philbrick 1993; Potamogetonaceae: Kaplan et al. 2013; Ruppia: Talavera et al. 1993).

\begin{tabular}{|c|c|c|c|c|c|c|c|c|}
\hline \multirow[t]{2}{*}{ Order } & \multirow[t]{2}{*}{ Family } & \multirow[t]{2}{*}{ Species } & \multirow{2}{*}{$\begin{array}{l}\text { Chromo- } \\
\text { some } \\
\text { number }\end{array}$} & \multicolumn{5}{|l|}{ Floristic region } \\
\hline & & & & Indian & Myanmar & Indo-china & $\begin{array}{l}\text { Sino- } \\
\text { Japanese }\end{array}$ & Others \\
\hline Acorales & Acoraceae & $\begin{array}{l}\text { Acorus } \\
\text { calamus L. }\end{array}$ & $2 n=24$ & $\begin{array}{l}\text { Subramanian } \\
\text { and Munian } \\
(1988)\end{array}$ & & & & $\begin{array}{l}\text { Chepinoga } \\
\text { et al. (2008) }\end{array}$ \\
\hline Acorales & Acoraceae & $\begin{array}{l}\text { Acorus } \\
\text { calamus L. }\end{array}$ & $2 n=35$ & & & & & $\begin{array}{l}\text { Krahulcová } \\
(2003)\end{array}$ \\
\hline Acorales & Acoraceae & $\begin{array}{l}\text { Acorus } \\
\text { calamus L. }\end{array}$ & $2 n=36$ & & & & & $\begin{array}{l}\text { Packer and } \\
\text { Ringius } \\
(1984)\end{array}$ \\
\hline Acorales & Acoraceae & $\begin{array}{l}\text { Acorus } \\
\text { calamus L. }\end{array}$ & $2 n=44$ & & & & $\begin{array}{l}\text { Wang et al. } \\
(2001)\end{array}$ & \\
\hline Acorales & Acoraceae & $\begin{array}{l}\text { Acorus } \\
\text { calamus L. }\end{array}$ & $2 n=45$ & $\begin{array}{l}\text { Ramachandran } \\
\text { (1978) }\end{array}$ & & & & \\
\hline Acorales & Acoraceae & $\begin{array}{l}\text { Acorus } \\
\text { calamus L. }\end{array}$ & $2 n=66$ & & & & $\begin{array}{l}\text { Wang et al. } \\
(2001)\end{array}$ & \\
\hline Acorales & Acoraceae & $\begin{array}{l}\text { Acorus } \\
\text { gramineus } \\
\text { Sol. ex Aiton }\end{array}$ & $2 n=24$ & & & & $\begin{array}{l}\text { Wang et al. } \\
\text { (2001) }\end{array}$ & \\
\hline Acorales & Araceae & $\begin{array}{l}\text { Cryptocoryne } \\
\text { crispatula } \\
\text { Engl. }\end{array}$ & $2 n=36$ & $\begin{array}{l}\text { Arends et al. } \\
(1982)\end{array}$ & & & & \\
\hline Acorales & Araceae & $\begin{array}{l}\text { Cryptocoryne } \\
\text { crispatula } \\
\text { Engl. }\end{array}$ & $2 n=54$ & $\begin{array}{l}\text { Jacobsen } \\
(1977)\end{array}$ & & & & \\
\hline Acorales & Araceae & $\begin{array}{l}\text { Cryptocoryne } \\
\text { cruddasiana } \\
\text { Prain }\end{array}$ & $\mathrm{n} / \mathrm{a}$ & & & & & \\
\hline Acorales & Araceae & $\begin{array}{l}\text { Pistia } \\
\text { stratiotes L. }\end{array}$ & $2 n=28$ & $\begin{array}{l}\text { Ramachandran } \\
(1978) \text {, } \\
\text { Subramanian } \\
\text { and Munian } \\
(1988)\end{array}$ & & & & \\
\hline
\end{tabular}




\begin{tabular}{|c|c|c|c|c|c|c|c|}
\hline Acorales & Araceae & $\begin{array}{l}\text { Landoltia } \\
\text { punctata (G. } \\
\text { Mey.) Les \& } \\
\text { D.J. Crawford }\end{array}$ & $\mathrm{n} / \mathrm{a}$ & & & & \\
\hline Acorales & Araceae & $\begin{array}{l}\text { Lemna } \\
\text { aequinoctialis } \\
\text { Welw. }\end{array}$ & $2 n=40$ & $\begin{array}{l}\text { Urbanska- } \\
\text { Worytkiewicz } \\
\text { (1975) (L. } \\
\text { perpusilla Torr.) }\end{array}$ & & $\begin{array}{l}\text { Beppu et al. } \\
\text { (1985) }\end{array}$ & \\
\hline Acorales & Araceae & $\begin{array}{l}\text { Lemna } \\
\text { trisulca L. }\end{array}$ & $2 n=20$ & & & & $\begin{array}{l}\text { Urbanska- } \\
\text { Worytkiewicz } \\
(1975)\end{array}$ \\
\hline Acorales & Araceae & $\begin{array}{l}\text { Lemna } \\
\text { trisulca L. }\end{array}$ & $2 n=40$ & & & & $\begin{array}{l}\text { Urbanska- } \\
\text { Worytkiewicz } \\
(1975)\end{array}$ \\
\hline Acorales & Araceae & $\begin{array}{l}\text { Lemna } \\
\text { trisulca L. }\end{array}$ & $2 n=60$ & & & & $\begin{array}{l}\text { Urbanska- } \\
\text { Worytkiewicz } \\
\text { (1975); Löve } \\
\text { and Löve } \\
(1981)\end{array}$ \\
\hline Acorales & Araceae & $\begin{array}{l}\text { Lemna } \\
\text { trisulca L. }\end{array}$ & $2 n=80$ & & & & $\begin{array}{l}\text { Urbanska- } \\
\text { Worytkiewicz } \\
(1975)\end{array}$ \\
\hline Acorales & Araceae & $\begin{array}{l}\text { Spirodela } \\
\text { polyrrhiza (L.) } \\
\text { Schleid. }\end{array}$ & $2 n=40$ & & & & $\begin{array}{l}\text { Löve and } \\
\text { Löve (1981), } \\
\text { Al-Bermani } \\
\text { et al. (1993) }\end{array}$ \\
\hline Acorales & Araceae & $\begin{array}{l}\text { Spirodela } \\
\text { polyrrhiza (L.) } \\
\text { Schleid. }\end{array}$ & $2 n=42$ & & & & $\begin{array}{l}\text { Chepinoga } \\
\text { et al. (2008) }\end{array}$ \\
\hline Acorales & Araceae & $\begin{array}{l}\text { Spirodela } \\
\text { polyrrhiza (L.) } \\
\text { Schleid. }\end{array}$ & $2 n=80$ & & & & $\begin{array}{l}\text { Geber and } \\
\text { Schweizer } \\
(1988)\end{array}$ \\
\hline Acorales & Araceae & $\begin{array}{l}\text { Wolffia } \\
\text { globosa } \\
\text { (Roxb.) } \\
\text { Hartog \&Plas }\end{array}$ & $\mathrm{n} / \mathrm{a}$ & & & & \\
\hline Alismatales & Alismataceae & $\begin{array}{l}\text { Alisma } \\
\text { plantago- } \\
\text { aquatica L. }\end{array}$ & $2 n=14$ & $\begin{array}{l}\text { Mehra and } \\
\text { Pandita (1984) }\end{array}$ & & $\begin{array}{l}\text { Wang et al. } \\
\text { (1987); } \\
\text { Uchiyama } \\
\text { (1989) (var. } \\
\text { orientale } \\
\text { Samuel) }\end{array}$ & \\
\hline Alismatales & Alismataceae & $\begin{array}{l}\text { Caldesia } \\
\text { parnassifolia } \\
\text { (Bassi ex L.) } \\
\text { Parl. }\end{array}$ & $\mathrm{n} / \mathrm{a}$ & & & & \\
\hline Alismatales & Alismataceae & $\begin{array}{l}\text { Limnocharis } \\
\text { flava (L.) } \\
\text { Buchenau }\end{array}$ & $2 n=20$ & & $\begin{array}{l}\text { This } \\
\text { study }\end{array}$ & $\begin{array}{l}\text { Uchiyama } \\
\text { (1989) }\end{array}$ & $\begin{array}{l}\text { Davidse } \\
(1981), \\
\text { Forni- } \\
\text { Martins and } \\
\text { Calligaris } \\
(2002)\end{array}$ \\
\hline Alismatales & Alismataceae & $\begin{array}{l}\text { Sagittaria } \\
\text { trifolia L. }\end{array}$ & $2 n=22$ & & $\begin{array}{l}\text { This } \\
\text { study }\end{array}$ & $\begin{array}{l}\text { Uchiyama } \\
\text { (1989); (var. } \\
\text { edulis (Sieb.) } \\
\text { Ohwi) }\end{array}$ & \\
\hline Alismatales & $\begin{array}{l}\text { Hydrocharita- } \\
\text { ceae }\end{array}$ & $\begin{array}{l}\text { Blyxa aubertii } \\
\text { Rich. }\end{array}$ & $2 n=40$ & & & $\begin{array}{l}\text { Uchiyama } \\
\text { (1989) }\end{array}$ & \\
\hline Alismatales & $\begin{array}{l}\text { Hydrocharita- } \\
\text { ceae }\end{array}$ & $\begin{array}{l}\text { Blyxa } \\
\text { echinosperma } \\
\text { (C.B. Clarke) } \\
\text { Hook. f. }\end{array}$ & $2 n=42$ & & & Wang (1986) & \\
\hline Alismatales & $\begin{array}{l}\text { Hydrocharita- } \\
\text { ceae }\end{array}$ & $\begin{array}{l}\text { Blyxa } \\
\text { echinosperma } \\
\text { (C.B. Clarke) } \\
\text { Hook. f. }\end{array}$ & $2 n=74$ & & & $\begin{array}{l}\text { Uchiyama } \\
\text { (1989) }\end{array}$ & \\
\hline
\end{tabular}




\begin{tabular}{|c|c|c|c|c|c|c|c|}
\hline Alismatales & $\begin{array}{l}\text { Hydrocharita- } \\
\text { ceae }\end{array}$ & $\begin{array}{l}\text { Blyxa } \\
\text { japonica } \\
\text { (Miq.) Maxim. } \\
\text { ex Asch. \& } \\
\text { Gürke }\end{array}$ & $2 n=42$ & & & $\begin{array}{l}\text { Harada } \\
(1956)\end{array}$ & \\
\hline Alismatales & $\begin{array}{l}\text { Hydrocharita- } \\
\text { ceae }\end{array}$ & $\begin{array}{l}\text { Blyxa } \\
\text { japonica } \\
\text { (Miq.) Maxim. } \\
\text { ex Asch. \& } \\
\text { Gürke }\end{array}$ & $2 n=72$ & & & $\begin{array}{l}\text { Uchiyama } \\
\text { (1989) }\end{array}$ & \\
\hline Alismatales & $\begin{array}{l}\text { Hydrocharita- } \\
\text { ceae }\end{array}$ & $\begin{array}{l}\text { Egeria densa } \\
\text { (Planch.) } \\
\text { Casp. }\end{array}$ & $2 n=46$ & & & $\begin{array}{l}\text { Uchiyama } \\
(1989), \\
\text { Nakata and } \\
\text { Nagai (1998) }\end{array}$ & \\
\hline Alismatales & $\begin{array}{l}\text { Hydrocharita- } \\
\text { ceae }\end{array}$ & $\begin{array}{l}\text { Egeria densa } \\
\text { (Planch.) } \\
\text { Casp. }\end{array}$ & $2 n=48$ & & & & $\begin{array}{l}\text { Löve and } \\
\text { Löve (1961) }\end{array}$ \\
\hline Alismatales & $\begin{array}{l}\text { Hydrocharita- } \\
\text { ceae }\end{array}$ & $\begin{array}{l}\text { Elodea } \\
\text { nuttallii } \\
\text { (Planch.) H. } \\
\text { St. John }\end{array}$ & $2 n=48$ & & & & $\begin{array}{l}\text { Simpson } \\
(1986)\end{array}$ \\
\hline Alismatales & $\begin{array}{l}\text { Hydrocharita- } \\
\text { ceae }\end{array}$ & $\begin{array}{l}\text { Hydrilla } \\
\text { verticillata (L. } \\
\text { f.) Royle }\end{array}$ & $2 n=16$ & $\begin{array}{l}\text { Chaudhuri and } \\
\text { Sharma (1978), } \\
\text { Pandita and } \\
\text { Mehra (1984) }\end{array}$ & & $\begin{array}{l}\text { Wang (1986), } \\
\text { Uchiyama } \\
\text { (1989), } \\
\text { Langeland et } \\
\text { al. (1992) }\end{array}$ & $\begin{array}{l}\text { Langeland et } \\
\text { al. (1992) }\end{array}$ \\
\hline Alismatales & $\begin{array}{l}\text { Hydrocharita- } \\
\text { ceae }\end{array}$ & $\begin{array}{l}\text { Hydrilla } \\
\text { verticillata (L. } \\
\text { f.) Royle }\end{array}$ & $2 n=24$ & $\begin{array}{l}\text { Chaudhuri and } \\
\text { Sharma (1978) }\end{array}$ & & $\begin{array}{l}\text { Langeland et } \\
\text { al. (1992), } \\
\text { Nakata and } \\
\text { Nagai (1998) }\end{array}$ & $\begin{array}{l}\text { Langeland et } \\
\text { al. (1992) }\end{array}$ \\
\hline Alismatales & $\begin{array}{l}\text { Hydrocharita- } \\
\text { ceae }\end{array}$ & $\begin{array}{l}\text { Hydrilla } \\
\text { verticillata (L. } \\
\text { f.) Royle }\end{array}$ & $2 n=32$ & & & $\begin{array}{l}\text { Langeland et } \\
\text { al. (1992) }\end{array}$ & $\begin{array}{l}\text { Langeland et } \\
\text { al. (1992) }\end{array}$ \\
\hline Alismatales & $\begin{array}{l}\text { Hydrocharita- } \\
\text { ceae }\end{array}$ & $\begin{array}{l}\text { Hydrocharis } \\
\text { dubia (Blume) } \\
\text { Backer }\end{array}$ & $2 n=16$ & $\begin{array}{l}\text { Pandita and } \\
\text { Mehra (1984) }\end{array}$ & & $\begin{array}{l}\text { Uchiyama } \\
\text { (1989) }\end{array}$ & \\
\hline Alismatales & $\begin{array}{l}\text { Hydrocharita- } \\
\text { ceae }\end{array}$ & $\begin{array}{l}\text { Najas } \\
\text { graminea } \\
\text { Delile }\end{array}$ & $2 n=12$ & & & $\begin{array}{l}\text { You et al. } \\
(1991)\end{array}$ & \\
\hline Alismatales & $\begin{array}{l}\text { Hydrocharita- } \\
\text { ceae }\end{array}$ & $\begin{array}{l}\text { Najas } \\
\text { graminea } \\
\text { Delile }\end{array}$ & $2 n=24$ & & & $\begin{array}{l}\text { Wang (1985), } \\
\text { Uchiyama } \\
\text { (1989) }\end{array}$ & \\
\hline Alismatales & $\begin{array}{l}\text { Hydrocharita- } \\
\text { ceae }\end{array}$ & $\begin{array}{l}\text { Najas } \\
\text { graminea } \\
\text { Delile }\end{array}$ & $2 n=36$ & & & $\begin{array}{l}\text { Uchiyama } \\
\text { (1989) }\end{array}$ & \\
\hline Alismatales & $\begin{array}{l}\text { Hydrocharita- } \\
\text { ceae }\end{array}$ & $\begin{array}{l}\text { Najas indica } \\
\text { (Willd.) } \\
\text { Cham. }\end{array}$ & $\mathrm{n} / \mathrm{a}$ & & & & \\
\hline Alismatales & $\begin{array}{l}\text { Hydrocharita- } \\
\text { ceae }\end{array}$ & $\begin{array}{l}\text { Najas marina } \\
\mathrm{L} .\end{array}$ & $2 n=12$ & & & $\begin{array}{l}\text { Wang (1985), } \\
\text { Uchiyama } \\
\text { (1989) }\end{array}$ & \\
\hline Alismatales & $\begin{array}{l}\text { Hydrocharita- } \\
\text { ceae }\end{array}$ & $\begin{array}{l}\text { Najas marina } \\
\mathrm{L} .\end{array}$ & $2 n=24$ & & & & $\begin{array}{l}\text { Viinikka et } \\
\text { al. (2008) }\end{array}$ \\
\hline Alismatales & $\begin{array}{l}\text { Hydrocharita- } \\
\text { ceae }\end{array}$ & $\begin{array}{l}\text { Najas tenuis } \\
\text { Magnus }\end{array}$ & $2 n=24$ & & $\begin{array}{l}\text { Ito et al. } \\
\text { (2014b) }\end{array}$ & & \\
\hline Alismatales & $\begin{array}{l}\text { Hydrocharita- } \\
\text { ceae }\end{array}$ & $\begin{array}{l}\text { Nechamandra } \\
\text { alternifolia } \\
\text { (Roxb.) } \\
\text { Thwaites }\end{array}$ & $2 n=16$ & $\begin{array}{l}\text { Sharma and } \\
\text { Chatterjee } \\
(1967)\end{array}$ & $\begin{array}{l}\text { Ito et al. } \\
(2009)\end{array}$ & & \\
\hline Alismatales & $\begin{array}{l}\text { Hydrocharita- } \\
\text { ceae }\end{array}$ & $\begin{array}{l}\text { Ottelia } \\
\text { alismoides } \\
\text { (L.) Pers. }\end{array}$ & $2 n=44$ & & & $\begin{array}{l}\text { Harada } \\
\text { (1956), } \\
\text { Uchiyama } \\
(1989)\end{array}$ & \\
\hline Alismatales & $\begin{array}{l}\text { Hydrocharita- } \\
\text { ceae }\end{array}$ & $\begin{array}{l}\text { Ottelia } \\
\text { alismoides } \\
\text { (L.) Pers. }\end{array}$ & $2 n=66$ & $\begin{array}{l}\text { Chaudhuri and } \\
\text { Sharma (1978) }\end{array}$ & & & \\
\hline
\end{tabular}




\begin{tabular}{|c|c|c|c|c|c|c|c|c|}
\hline Alismatales & $\begin{array}{l}\text { Hydrocharita- } \\
\text { ceae }\end{array}$ & $\begin{array}{l}\text { Ottelia } \\
\text { alismoides } \\
\text { (L.) Pers. }\end{array}$ & $2 n=68$ & $\begin{array}{l}\text { Chaudhuri and } \\
\text { Sharma (1978) }\end{array}$ & & & & \\
\hline Alismatales & $\begin{array}{l}\text { Hydrocharita- } \\
\text { ceae }\end{array}$ & $\begin{array}{l}\text { Ottelia } \\
\text { cordata } \\
\text { (Wall.) Dandy }\end{array}$ & $\mathrm{n} / \mathrm{a}$ & & & & & \\
\hline Alismatales & $\begin{array}{l}\text { Hydrocharita- } \\
\text { ceae }\end{array}$ & $\begin{array}{l}\text { Vallisneria } \\
\text { spiralis L. }\end{array}$ & $2 n=20$ & & & & Wang (1986) & \\
\hline Alismatales & $\begin{array}{l}\text { Hydrocharita- } \\
\text { ceae }\end{array}$ & $\begin{array}{l}\text { Vallisneria } \\
\text { spiralis L. }\end{array}$ & $2 n=24$ & $\begin{array}{l}\text { Chaudhuri and } \\
\text { Sharma (1978) }\end{array}$ & & & & \\
\hline Alismatales & $\begin{array}{l}\text { Hydrocharita- } \\
\text { ceae }\end{array}$ & $\begin{array}{l}\text { Vallisneria } \\
\text { spiralis L. }\end{array}$ & $2 n=30$ & $\begin{array}{l}\text { Chaudhuri and } \\
\text { Sharma (1978) }\end{array}$ & & & & \\
\hline Alismatales & $\begin{array}{l}\text { Hydrocharita- } \\
\text { ceae }\end{array}$ & $\begin{array}{l}\text { Vallisneria } \\
\text { spiralis L. }\end{array}$ & $2 n=40$ & $\begin{array}{l}\text { Chaudhuri and } \\
\text { Sharma (1978), } \\
\text { Sarkar et al. } \\
(1980)\end{array}$ & & & & \\
\hline Alismatales & $\begin{array}{l}\text { Aponogetona- } \\
\text { ceae }\end{array}$ & $\begin{array}{l}\text { Aponogeton } \\
\text { lakhonensis } \\
\text { A. Camus }\end{array}$ & $\mathrm{n} / \mathrm{a}$ & & & & & \\
\hline Alismatales & $\begin{array}{l}\text { Potamogetona- } \\
\text { ceae }\end{array}$ & $\begin{array}{l}\text { Potamogeton } \\
\text { crispus L. }\end{array}$ & $2 n=52$ & & & & $\begin{array}{l}\text { Kaplan et al. } \\
(2013)\end{array}$ & $\begin{array}{l}\text { Kaplan et al. } \\
(2013)\end{array}$ \\
\hline Alismatales & $\begin{array}{l}\text { Potamogetona- } \\
\text { ceae }\end{array}$ & $\begin{array}{l}\text { Potamogeton } \\
\text { crispus L. }\end{array}$ & $2 n=56$ & & & & $\begin{array}{l}\text { Nakata and } \\
\text { Nagai (1998) }\end{array}$ & \\
\hline Alismatales & $\begin{array}{l}\text { Potamogetona- } \\
\text { ceae }\end{array}$ & $\begin{array}{l}\text { Potamogeton } \\
\text { distinctus A. } \\
\text { Benn. }\end{array}$ & $2 n=52$ & & & & $\begin{array}{l}\text { Kaplan et al. } \\
(2013)\end{array}$ & \\
\hline Alismatales & $\begin{array}{l}\text { Potamogetona- } \\
\text { ceae }\end{array}$ & $\begin{array}{l}\text { Potamogeton } \\
\text { distinctus A. } \\
\text { Benn. } \times \text { P. } \\
\text { nodosus Poir. }\end{array}$ & $2 n=52$ & & $\begin{array}{l}\text { This } \\
\text { study }\end{array}$ & & & \\
\hline Alismatales & $\begin{array}{l}\text { Potamogetona- } \\
\text { ceae }\end{array}$ & $\begin{array}{l}\text { Potamogeton } \\
\text { maackianus A } \\
\text { Benn. }\end{array}$ & $2 n=52$ & & & & $\begin{array}{l}\text { Kaplan et al. } \\
(2013)\end{array}$ & \\
\hline Alismatales & $\begin{array}{l}\text { Potamogetona- } \\
\text { ceae }\end{array}$ & $\begin{array}{l}\text { Potamogeton } \\
\text { maackianus A } \\
\text { Benn. }\end{array}$ & $2 n=56$ & & & $\begin{array}{l}\text { Kaplan et al. } \\
(2013)\end{array}$ & $\begin{array}{l}\text { Uchiyama } \\
(1989), \\
\text { Kaplan et al. } \\
(2013)\end{array}$ & \\
\hline Alismatales & $\begin{array}{l}\text { Potamogetona- } \\
\text { ceae }\end{array}$ & $\begin{array}{l}\text { Potamogeton } \\
\times \text { malainoides } \\
\text { Miki }\end{array}$ & $2 n=52$ & & & & $\begin{array}{l}\text { Kaplan et al. } \\
(2013)\end{array}$ & \\
\hline Alismatales & $\begin{array}{l}\text { Potamogetona- } \\
\text { ceae }\end{array}$ & $\begin{array}{l}\text { Potamogeton } \\
\text { lucens L. }\end{array}$ & $2 n=52$ & & & & $\begin{array}{l}\text { Kaplan et al. } \\
(2013)\end{array}$ & $\begin{array}{l}\text { Kaplan et al. } \\
(2013)\end{array}$ \\
\hline Alismatales & $\begin{array}{l}\text { Potamogetona- } \\
\text { ceae }\end{array}$ & $\begin{array}{l}\text { Potamogeton } \\
\text { nodosus Poir. }\end{array}$ & $2 n=52$ & & & & & $\begin{array}{l}\text { Kaplan et al. } \\
(2013)\end{array}$ \\
\hline Alismatales & $\begin{array}{l}\text { Potamogetona- } \\
\text { ceae }\end{array}$ & $\begin{array}{l}\text { Potamogeton } \\
\text { octandrus } \\
\text { Poir. }\end{array}$ & $2 n=28$ & & & & $\begin{array}{l}\text { Uchiyama } \\
\text { (1989), } \\
\text { Nakata and } \\
\text { Nagai (1998), } \\
\text { Kaplan et al. } \\
(2013)\end{array}$ & \\
\hline Alismatales & $\begin{array}{l}\text { Potamogetona- } \\
\text { ceae }\end{array}$ & $\begin{array}{l}\text { Potamogeton } \\
\text { wrightii } \\
\text { Morong }\end{array}$ & $2 n=52$ & & & & $\begin{array}{l}\text { Kaplan et al. } \\
(2013)\end{array}$ & \\
\hline Alismatales & $\begin{array}{l}\text { Potamogetona- } \\
\text { ceae }\end{array}$ & $\begin{array}{l}\text { Stuckenia } \\
\text { pectinata (L.) } \\
\text { Börner }\end{array}$ & $2 n=78$ & $\begin{array}{l}\text { Kaplan et al. } \\
(2013)\end{array}$ & & & & $\begin{array}{l}\text { Kaplan et al. } \\
(2013)\end{array}$ \\
\hline Alismatales & $\begin{array}{l}\text { Potamogetona- } \\
\text { ceae }\end{array}$ & $\begin{array}{l}\text { Stuckenia } \\
\text { pectinata (L.) } \\
\text { Börner }\end{array}$ & $2 n=84$ & & & & $\begin{array}{l}\text { Uchiyama } \\
\text { (1989) }\end{array}$ & \\
\hline \multirow[t]{2}{*}{ Alismatales } & Ruppiaceae & $\begin{array}{l}\text { Ruppia } \\
\text { maritima L. }\end{array}$ & $2 n=20$ & Ito et al. (2010) & & Ito et al. (2010) & $\begin{array}{l}\text { Ito et al. } \\
(2010)\end{array}$ & $\begin{array}{l}\text { Van } \\
\text { Vierssen et } \\
\text { al. (1981) }\end{array}$ \\
\hline & & $\begin{array}{l}\text { Ruppia } \\
\text { maritima L. }\end{array}$ & $2 n=40$ & & & & $\begin{array}{l}\text { Harada } \\
(1956), \text { Ito et } \\
\text { al. }(2010)\end{array}$ & $\begin{array}{l}\text { Ito et al. } \\
(2010)\end{array}$ \\
\hline
\end{tabular}




\begin{tabular}{|c|c|c|c|c|c|c|c|}
\hline Asparagales & Amaryllidaceae & $\begin{array}{l}\text { Crinum } \\
\text { thaianum J. } \\
\text { Schul. }\end{array}$ & $\mathrm{n} / \mathrm{a}$ & & & & \\
\hline Commelinales & Pontederiaceae & $\begin{array}{l}\text { Eichhornia } \\
\text { crassipes } \\
\text { (Mart.) Solms }\end{array}$ & $2 n=32$ & & & & $\begin{array}{l}\text { Pedrosa et } \\
\text { al. (1999) }\end{array}$ \\
\hline Commelinales & Pontederiaceae & $\begin{array}{l}\text { Monochoria } \\
\text { hastata (L.) } \\
\text { Solms }\end{array}$ & $2 n=28$ & $\begin{array}{l}\text { Patwary et al. } \\
\text { (1989) }\end{array}$ & & & \\
\hline Commelinales & Pontederiaceae & $\begin{array}{l}\text { Monochoria } \\
\text { hastata }(\mathrm{L} .) \\
\text { Solms }\end{array}$ & $2 n=80$ & $\begin{array}{l}\text { Patwary et al. } \\
\text { (1989) }\end{array}$ & & & \\
\hline Commelinales & Pontederiaceae & $\begin{array}{l}\text { Monochoria } \\
\text { vaginalis } \\
\text { (Burm.f.) C. } \\
\text { Presl ex } \\
\text { Kunth }\end{array}$ & $2 n=24$ & $\begin{array}{l}\text { Christopher } \\
\text { (1983) (var. } \\
\text { plantaginea } \\
\text { (Roxb.) } \\
\text { Solms);Patwary } \\
\text { et al. (1989) }\end{array}$ & & & \\
\hline Commelinales & Pontederiaceae & $\begin{array}{l}\text { Monochoria } \\
\text { vaginalis } \\
\text { (Burm.f.) C. } \\
\text { Presl ex } \\
\text { Kunth }\end{array}$ & $2 n=48$ & & $\begin{array}{l}\text { Wang and } \\
\text { Kusanagi } \\
\text { (1996) (var. } \\
\text { angustifolia } \\
\text { G.X.Wang) }\end{array}$ & & \\
\hline Commelinales & Pontederiaceae & $\begin{array}{l}\text { Monochoria } \\
\text { vaginalis } \\
\text { (Burm.f.) C. } \\
\text { Presl ex } \\
\text { Kunth }\end{array}$ & $2 n=52$ & $\begin{array}{l}\text { Christopher } \\
\text { (1983), Patwary } \\
\text { et al. (1989) }\end{array}$ & & $\begin{array}{l}\text { Wang and } \\
\text { Kusanagi } \\
(1996)\end{array}$ & \\
\hline Commelinales & Typhaceae & $\begin{array}{l}\text { Typha } \\
\text { angustifolia L. }\end{array}$ & $2 n=30$ & & & & $\begin{array}{l}\text { Löve and } \\
\text { Löve (1981) }\end{array}$ \\
\hline Poales & Eriocaulaceae & $\begin{array}{l}\text { Eriocaulon } \\
\text { setaceum L. }\end{array}$ & $\mathrm{n} / \mathrm{a}$ & & & & \\
\hline
\end{tabular}

\section{Discussion}

Of 45 non-hybrid aquatic monocots and two interspecific hybrids among them, more than two thirds have no chromosome variation. Meanwhile, the following nine species have infra-specific chromosome variation, i.e., Acorus calamus, Cryptocoryne crispatula, Blyxa echinosperma, Hydrilla verticillata, Najas graminea, Ottelia alismoides, Vallisneria spiralis, Monochoria hastata, and M. vaginalis (Table 1). Among the cytologically variable aquatic monocots are $A$. calamus, $O$. alismoides, $V$. spiralis, and $M$. vaginalis, for which unique chromosome counts are obtained from each floristic region. Myanmar is known as including borders among the Indian, the Indochina, and the Sino-Japanese floristic regions (Tanaka 2010), yet in the aquatic flora, it is unknown which flora is more influenced. Future research with a focus on such species will address this issue.

Potamogeton is known as having numerous inter-specific hybrids, and each parental combination is varied from intra-ploidy crosses to inter-ploidy ones (Kaplan et al. 2013). The present study revealed $P$. distinctus $\times P$. nodosus as another intra-ploidy hybrid of Potamogeton at tetraploid level. 


\section{Acknowledgements}

Thanks are due to the following personnel in Myanmar for their help in arranging our field work: U. Htun Paw Oo, Ex-director of Nature and Wildlife Conservation Division; U. Soe Win Hlaing, Ex-director General, Forest Department, Ministry of Environmental Conservation and Forestry. We also thank Prof. T. Koyama, director of the Kochi Prefectural Makino Botanical Garden (Japan), who initiated the Myanmar-Japanese cooperative program to inventory the plants of Myanmar, and gave us the opportunities to study the Myanmar plants, Prof. J. Murata, curator of the University of Tokyo (Japan), who principally organizes the Myanmar-Japanese inventory project, and Dr. T. Ohi-Toma, assistant professor of the University of Tokyo (Japan), who helps the first author to use the facilities of the botanical gardens, the university of Tokyo.

\section{Author contributions}

Conceived and designed the study: YI NT. Collected the samples in the field: YI NT. Performed the chromosome observation: YI. Analyzed the data: YI. Wrote the paper: YI. Corrected and revised manuscript: NT.

\section{References}

- $\quad$ Al-Bermani AK, Al-Shammary KI, Bailey JP, Gornall RJ (1993) Contribution to a cytological catalogue of the British and Irish flora 3. Watsonia 19: 269-271. URL: http://archive.bsbi.org.uk/Wats19p269.pdf

- $\quad$ Arends JC, Bastmeijer JD, Jacobsen N (1982) Chromosome numbers and taxonomy in Cryptocoryne (Araceae). II. Nordic Journal of Botany 2 (5): 453-463. DOI: 10.1111/j.1756-1051.1982.tb01208.x

- $\quad$ Beppu T, Yanase D, Nobuchi T, Murata G (1985) A revision of Lemna paucicostata group in Japan. Acta Phytotaxonomica et Geobotanica 36: 45-58. URL: http:// ci.nii.ac.jp/els/110003760235.pdf? id=ART0004972021\&type $=$ pdf\&lang $=$ jp\&host $=$ cinii\&order no=\&ppv type=0\&lang $s w=\& n o=1396$

- Chaudhuri JB, Sharma A (1978) Cytological studies on three aquatic members of Hydrocharitaceae in relation to their morphological and ecological characteristics. Cytologia 43 (1): 1-19. DOI: 10.1508/cytologia.43.1

- Chepinoga VV, Gnutikov AA, Enushchenko IV, Chepinoga AV (2008) IAPT/IOPB chromosome data 6. Taxon 57: 1267-1268.

- Christopher J (1983) Cytology of Monochoria vaginalis complex Presl. Cytologia 48 (3): 627-631. DOI: 10.1508/cytologia.48.627

- Cook CD (1996) Aquatic and Wetland Plants of India: A reference book and identification manual for the vascular plants found in permanent or seasonal fresh water in the subcontinent of India south of the Himalayas. Oxford University Press, Oxford, $385 \mathrm{pp}$. 
- Davidse G (1981) Chromosome numbers of miscellaneous angiosperms. Annals of the Missouri Botanical Garden 68 (1): 222. DOI: 10.2307/2398824

- Fedorov A (1969) Chromosome Numbers of Flowering Plants. Academy of Sciences of USSR, Leningrad, $926 \mathrm{pp}$.

- $\quad$ Forni-Martins ER, Calligaris KP (2002) Chromosomal studies on Neotropical Limnocharitaceae (Alismatales). Aquatic Botany 74 (1): 33-41. DOI: $10.1016 /$ s0304-3770(02)00038-4

- Geber G, Schweizer D (1988) Cytochemical heterochromatin differentiation in Sinapis alba (Cruciferae) using a simple air-drying technique for producing chromosome spreads. Plant Systematics and Evolution 158: 97-106. DOI: 10.1007/ bf00936336

- Harada I (1956) Cytological studies in Helobiae: I. Chromosome idiograms and a list of chromosome numbers in seven families. Cytologia 21 (3): 306-328. DOI: 10.1508/cytologia.21.306

- Ito Y, Barfod A (2014) An updated checklist of aquatic plants of Myanmar and Thailand. Biodiversity Data Journal 2: e1019. DOI: 10.3897/bdj.2.e1019

- Ito Y, Ohi-Toma T, Murata J, Tanaka N (2010) Hybridization and polyploidy of an aquatic plant, Ruppia (Ruppiaceae), inferred from plastid and nuclear DNA phylogenies. American Journal of Botany 97 (7): 1156-1167. DOI: 10.3732/ ajb.0900168

- Ito Y, Ohi-Toma T, Tanaka N, Murata J (2009) New or noteworthy plant collections from Myanmar (3) Caldesia parnassifolia, Nechamandra alternifolia, Potamogeton maackianus and P. octandrus. The Journal of Japanese Botany 84 (6): 321-329.

- Ito Y, Tanaka N, Rachun P, Tanaka N (2014a) DNA barcoding reveals a new record of Potamogeton distinctus (Potamogetonaceae) and its natural hybrids, $P$. distinctus $\times P$. nodosus and $P$. distinctus $\times P$. wrightii $(P . \times$ malainoides $)$ from Myanmar. Biodiversity Data Journal 2: e1073. DOI: $10.3897 / \mathrm{bdj} .2 . e 1073$

- Ito Y, Ohi-Toma T, Tanaka N, Tanaka N, Murata J (2014b) New or noteworthy plant collections from Myanmar (8) Blyxa aubertii var. echinosperma, Lemna trisulca, and Najas tenuis. APG: Acta Phytotaxonomica et Geobotanica 65 (2): xx-xx.

- Jacobsen N (1977) Chromosome numbers and taxonomy in Cryptocoryne (Araceae). Botaniska Notiser 130: 71-87.

- Kaplan Z, Jarolímová V, Fehrer J (2013) Revision of chromosome numbers of Potamogetonaceae: a new basis for taxonomic and evolutionary implications. Preslia 85: 421-487. URL: http://www.preslia.cz/P134Kaplan.pdf

- Krahulcová A (2003) Chromosome numbers in selected monocotyledons (Czech Republic, Hungary, and Slovakia). Preslia 75: 97-113. URL: http://www.preslia.cz/ P032CKra.pdf

- Kress WJ, De Filipps RA, Farr E, Kyi DY (2003) A Checklist of the Trees, Shrubs, Herbs, and Climbers of Union of Myanmar. Contributions from the United States National Herbarium. National Museum of Natural History, Washington D.C., 1-590 pp.

- $\quad$ Langeland KA, Shilling DG, Carter JL, Laroche FB, Steward KK, Madiera PT (1992) Chromosome morphology and number in various populations of Hydrilla verticillata (L.f.) Royle. Aquatic Botany 42 (3): 253-263. DOI: 10.1016/0304-3770(92)90026-f 
- Les H, Philbrick T (1993) Studies of hybridization and chromosome number variation in aquatic angiosperms: evolutionary implications. Aquatic Botany 44: 181-228. DOI: 10.1016/0304-3770(93)90071-4

- Löve A, Löve D (1961) Chromosome numbers of central and northwest European plant species. Opera Botanica 5: 1-581.

- $\quad$ Löve A, Löve D (1981) Chromosome number reports LXXII. Taxon 30: 699-701.

- Mehra PN, Pandita TK (1984) Cytological studies of some helobiales of Kashmir Himalayas. I. Family Alismataceae. Cytologia 49 (2): 295-304. DOI: 10.1508/ cytologia.49.295

- $\quad$ Nakata M, Nagai S (1998) Chromosome numbers of some aquatic plants collected in Toyama Prefecture. Toyama-no-Seibutsu 37: 1-6. [In Japanese].

- $\quad$ Packer J, Ringius G (1984) The distribution and status of Acorus (Araceae) in Canada. Canadian Journal of Botany 62 (11): 2248-2252. DOI: 10.1139/b84-305

- $\quad$ Pandita TK, Mehra PN (1984) Cytological studies of some helobiales of Kashmir Himalayas. II. Families: Butomaceae and Hydrocharitaceae. Cytologia 49 (2): 305-312. DOI: $10.1508 /$ cytologia.49.305

- $\quad$ Patwary M, Haque MM, Zaman MA (1989) Polyploidy in Monochoria hastata Solms, and M. vaginalis Prest. grown in Bangladesh. Cytologia 54 (3): 505-511. DOI: $10.1508 /$ cytologia.54.505

- $\quad$ Pedrosa A, Gitai J, Silva AEB, Felix LP, Guerra M (1999) Citogenética de angiospermas coletadas em Pernambuco - V. Acta Botanica Brasilica 13 (1): 49-60. [In Portuguese]. URL: http://www.scielo.br/pdf/abb/v13n1/v13n1a06.pdf

- $\quad$ Ramachandran K (1978) Cytological studies on south Indian araceae. Cytologia 43 (2): 289-303. DOI: 10.1508/cytologia.43.289

- $\quad$ Sanders R, Stuessy T, Rodriguez R (1983) Chromosome numbers from the flora of the Juan Fernandez islands. American Journal of Botany 70 (6): 799-810. DOI:

$10.2307 / 2442929$

- Sarkar A, Datta N, Chatterjee U (1980) Chromosome number reports LXVII. Taxon 29: 360-361.

- Sharma AK, Chatterjee T (1967) Cytotaxonomy of Helobiae with special reference to the mode of evolution. Cytologia 32 (2): 286-307. DOI: 10.1508/cytologia.32.286

- Simpson DA (1986) Taxonomy of Elodea Michx in the British Isles. Watsonia 16: 1-14. URL: http://archive.bsbi.org.uk/Wats16p1.pdf

- Stace C (2000) Cytology and cytogenetics as a fundamental taxonomic resource for the 20th and 21st centuries. Taxon 49 (3): 451. DOI: $10.2307 / 1224344$

- Subramanian D, Munian M (1988) Cytotaxonomical studies in South Indian Araceae. Cytologia 53 (1): 59-66. DOI: 10.1508/cytologia.53.59

- Talavera S, García-Murillo P, Herrera J (1993) Chromosome numbers and a new model for karyotype evolution in Ruppia L. (Ruppiaceae). Aquatic Botany 45 (1): 1-13. DOI: 10.1016/0304-3770(93)90048-2

- Tanaka N (2005) Plant inventory research: contributions to the flora of Myanmar. APG: Acta Phytotaxonomica et Geobotanica 56: 1-26. URL: http://ci.nii.ac.jp/ naid/110006318373

- Tanaka N (2010) Plant inventory research in Myanmar. Bunrui 10 (2): 139-149. [In Japanese]. URL: http://ci.nii.ac.jp/naid/110007681490 
- Tanaka N, Koyama T, Murata J (2006) The flowering plants of Mt. Popa, central of Myanmar - Results of Myanmar-Japanese joint expeditions 2000-2004. Makinoa New Series 5: 1-95.

- Uchiyama H (1989) Karyomorphological studies on some taxa of the Helobiae. Journal of Science, Hiroshima University, Series B, Division 2 (Botany) 22: 271-352.

- $\quad$ Urbanska-Worytkiewicz K (1975) Cytological variation within Lemna L. Aquatic Botany 1: 377-394. DOI: 10.1016/0304-3770(75)90038-8

- $\quad$ Van Dijk PP, Tordoff AW, Fellowes J, Lau M, Jinshuang M (2004) Indo-Burma. In: Mittermeier RA, Robles GP, Hoffmann M, Lamoreaux J, da Fonseca GA (Eds) Hotspots Revisited: Earth's Biologically Richest and Most Endangered Terrestrial Ecoregions. CEMEX, Agrupación Sierra Madre, 323-330 pp.

- Van Vierssen W, Van Wijk RJ, Van der Zee JR (1981) Some additional notes on the cytotaxonomy of Ruppia taxa in Western Europe. Aquatic Botany 11: 297-301. DOI: 10.1016/0304-3770(81)90067-x

- Viinikka Y, Agami M, Triest L (2008) A tetraploid cytotype of Najus marina L. Hereditas 106 (2): 289-291. DOI: 10.1111/j.1601-5223.1987.tb00262.x

- Wang G, Kusanagi T (1996) Cytotaxonomic analyses of the genus Monochoria in Asia. APG: Acta phytotaxonomica et geobotanica 47: 105-111. URL: http:// ci.nii.ac.jp/naid/110003758862

- Wang H, Li W, Gu Z, Chen Y (2001) Cytological study on Acorus L. in southwestern China, with some cytogeographical notes on A. calamus. Acta Botanica Sinica 43 (4): 354-358. [In Chinese]. URL: http://www.jipb.net/pubsoft/content/2/2013/ X000331(PS2).pdf

- Wang N (1985) A preliminary investigation and cytotaxonomic study on Najadaceae in China. Journal of Wuhan Botanical Research 3 (1): 29-44. [In Chinese]. URL: http://caod.oriprobe.com/articles/826892/

zhong guo ci zao ke zhi wu de chu bu diao cha ji xi bao xue fen lei ya.htm

- Wang N (1986) Chromosome numbers and karyotype analysis of 9 Species in Hydrocharitaceae. Acta Phytotaxonomica Sinica 24 (5): 370-375. URL: http:// www.plantsystematics.com/qikan/epaper/zhaiyao.asp?bsid=13947

- Wang Z, Sun X, Wang H (1987) Cytotaxonomic studies on Alisma L. from Hubei. Acta Phytotaxonomica Sinica 25 (4): 254-263. [In Chinese]. URL: http:// www.plantsystematics.com/qikan/epaper/zhaiyao.asp?bsid=14007

- You J, Sun X, Wang H (1991) A preliminary study on the polyploid series and cytogeography of Najas graminea. Acta Phytotaxonomica Sinica 29 (3): 230-234. [In Chinese]. URL: http://www.plantsystematics.com/qikan/epaper/zhaiyao.asp? $\underline{\text { bsid }=10769}$ 